renal involvement being the principal cause. It requires close monitoring and treatment. Renal biopsies can be important prognostic markers and guide best management.(2) Currently, there have been no significant randomised controlled trials detailing best management and hence treatment varies significantly. This study reviews practice in a national tertiary renal unit in Ireland over a fifteen-year period.

Aims 1. Identify children who had a renal biopsy.

2. Review Medical management, duration of therapy and follow up.

Methods This was a retrospective review between September 2001 to April 2017. Data was collated by computer and paper records, online haematological and radiological resources. This review excludes children managed in another tertiary centre in Ireland.

Results

The main outcomes were patient demographics, (i) Biopsy findings: Acute vs Chronic and staging classification, (ii) Drug Treatment and Duration (iii) Follow up: urinanalysis at interval time points, renal ultrasonography and renal function. Thirty children had a renal biopsy. 10 For persistent proteinuria, 2 for recurrent episodes, deranged renal function, mixed nephritic/nephrotic, IgA vs HSP respectively and 12 not classified.

$73 \%$ required medical treatment, the majority polypharmacy. When immunosuppressants were needed a combination of steroids and Mycophenolate Acid were most commonly used. $73 \%$ required medical treatment for over eighteen months with evidence of proteinuria often guiding duration.

Regular follow up included urinanalyis, renal bloods, ultrasonography and blood pressure measurement.

To standarise care nationally, a basic guideline was created to aid appropriate referral to our tertiary centre.

Conclusion HSP is rare but can cause significant morbidity. RCTs are required to determine best management and to standardize care nationally and internationally

\section{P559 A POSSIBLE PATHOGENIC RELATIONSHIP BETWEEN ENURESIS AND INNOCENT HEART MURMURS}

${ }^{1}$ Pietro Ferrara*, ${ }^{2}$ Federica Di Ruscio, ${ }^{2}$ Margherita Zona, ${ }^{2}$ Roberta Autuori, ${ }^{2}$ Alessandro Di Lucia, ${ }^{2}$ Flavia Dosa, ${ }^{2}$ Giulia Franceschini, ${ }^{2}$ Diletta Saitta, ${ }^{2}$ Michela Pulcino, ${ }^{1}$ Ester Del Vescovo, ${ }^{3}$ Massimo Pettoello-Mantovani. ${ }^{1}$ Institute of Pediatrics, Università Cattolica del Sacro Cuore, Rome, Italy; ${ }^{2}$ Campus Bio-Medico University, Rome, Italy; ${ }^{3}$ Department of Pediatrics, Scientific Institute Casa Sollievo della Sofferenza, University of Foggia, Foggia, Italy

\subsection{6/archdischild-2019-epa.893}

Objective The aim of this study is to evaluate the prevalence of innocent heart murmurs in children affected by nocturnal enuresis (NE). Specifically, for this purpose, a possible pathogenic relationship linking both conditions.

Design This study is an observational study.

Setting The study was characterized by a careful examination of patient's medical history and performing of physical examination, including the weight and height measurement. Mostly, it was a cardiological examination including monitoring patient's blood pressure and abnormal heart sounds: patent's foramen ovale, slight systolic impurity, mitral insufficiency, muffled heart sound, heart murmur, and impurity. The study was carried out in compliance with the Helsinki Declaration. Analyses were conducted using the Microsoft Excel 2011 (version 14.0.0) program. We used descriptive statistics to describe patients' information (age, gender, familiarity). Data were expressed as the mean \pm standard deviation and percentage. The $\chi^{2}$ test was used for categorical variables. The significance level was set at $p<0.05$.

Patients We are enrolling about 400 children (G1), 300 males and 100 females aged between 5 and 15 years, affected from NE referred to the Service of Pediatrics, 'Campus Bio-Medico' University Hospital of Rome, from September 2013 to January 2019 in the study. The control group is composed of about 400 children without NE (G2).

Main outcome measures Prevalence of innocent heart murmurs in children with MNE or N-MNE.

There isn't any study investigating the association of NE with innocent murmur in the literature.

Results The prevalence of heart innocent cardiac murmur appears to be significantly higher in children who suffer from NE. The results obtained are not definitive, because the study is ongoing.

Conclusions These findings made us acquaintance of the presence of possible underlying mechanisms which explain the association between higher prevalence of innocent heart murmur and enuresis, further studies are needed. A possible explanation of this relationship could be provided by heart increased secretion of atrial natriuretic peptide (ANP) and Btype natriuretic peptide (B-BNP). In particular, the murmur could be accentuated by hypervolemia, which in turn determines atrial and ventricular distention. This stimulates cardiomyocytes into producing ANP and B-BNP. ANP shares with BBNP a high degree of structural homology and a profile of diuretic, natriuretic and vasodilator activities and inhibition of the renin-angiotensin-aldosterone system.

\section{P560 URINARY SCREENING IN 6-12 YEARS SCHOOL CHILDREN IN ' MAKKAH \& AL-BAHA REGIONS' IN SAUDI ARABIA}

Mahmoud Rashad*. King Fahd Hospital, Al Baha, Saudi Arabia. Al Azher University, Cairo, Egypt

10.1136/archdischild-2019-epa.894

Introduction and aim of the work

Mass urinary screening is a useful tool to identify children with asymptomatic progressive renal diseases. Patients with renal disease have a variety of different clinical presentations, that are directly referred to the kidney or to extra renal clinical manifestation. Proteinuria as well as hematuria may be the only early signs of renal disease. UTI is very common in children with severe consequences on the kidney function leading to chronic kidney disease (CKD) and hypertension if left untreated. Hypercalciuria is the most common cause of urolithiasis in children.

The purpose of this study is to screen elementary school children in Makkah and Al-Baha region in Saudi Arabia, for prevalence of hematuria, proteinuria and hypercalciuria. The goal for screening of healthy individual or population for diseases is to reduce morbidity and mortality through early detection and treatment.

Subjects and methods This cross sectional study, approved by the Institutional Review Board Committees of Makkah and Al Baha, and was carried out between October 2016 and April 2017 on randomly selected 12352 children (including 9299 children from Makkah and 5053 children from Al Baha). Dipstick test was performed by a pediatrician. Children with abnormal urinary findings in the second screening were 
brought to the Department of Pediatrics (in KFH- Al Baha or Al Nour Hospital - Makkah) and subjected to: a detailed history, physical \& systemic examinations. Also more urinary, blood and ultrasound studies were done.

Results In Makkah region 1st screening shows that prevalence of hematuria was $0.7 \% \& 0.8 \%$, became by 2 nd screening $0.1 \% \& 0.1 \%$ in male and female, respectively, for proteinuria prevalence by 1 st screening was $1 \% \& 0.8 \%$ became by 2nd screening $0.1 \% \& 0.2 \%$ in male \& female respectively. The prevalence of hypercalciuria by 2 nd screening was $0.1 \%$ $\& 0.1 \%$ in male and female respectively, none of hematuria cases were hypercalciuric.

In Al Baha region prevalence of hematuria by 1 st screening was $0.7 \% \& 0.8 \%$, became by 2 nd screening $0.1 \%$ \& $0.2 \%$ in male \& female, respectively, for proteinuria prevalence by 1 st screening was $0.7 \%$ \& $0.8 \%$ became by 2 nd screening $0.2 \% \& 0.2 \%$ in male and female respectively. The prevalence of hypercalciuria by 2 nd screening $0.2 \% \& 0.2 \%$ in male $\&$ female respectively, and $67.6 \%$ of male hematuria cases, and $71.5 \%$ of female hematuria cases have hypercalciuria.

Conclusion Urinary screening would therefore not only help in early detection but also in the prevention of the deterioration of renal function later in life.

\section{P561 PSEUDOHYPOALDOSTERONISM SECONDARY TO URINARY TRACT INFECTION IN INFANTS}

Sultan Ceren Yıldırım*, Gamze Seval Şahin, Bahriye Atmış, İhsan Turan, Engin Melek, Aysun Karabay Bayazıt. Çukurova University, Adana, Turkey

\subsection{6/archdischild-2019-epa.895}

Hyponatremia with hyperkalemia in infancy is a rare but lifethreatening condition. In the first weeks of life, this scenario is usually associated with aldosterone deficiency due to congenital adrenal hyperplasia (CAH). Pseudohypoaldosteronism (PHA) should be considered in the differential diagnosis of $\mathrm{CAH}$ in infants with hyperkalemia, hyponatremia and metabolic acidosis. Urinary tract infections and/or urinary tract anomalies are the most common causes of type 1 secondary (transient) PHA. Adequate replacement with intravenous saline and antibiotic therapy is necessary to correct electrolyte levels and metabolic acidosis within 24-48 hours. Recognition of type 1 secondary PHA enables appropriate management, thus avoiding unnecessary investigations and treatment. Here, we presented five chidren that emphasized the clinical and biochemical properties of type 1 secondary PHA.

In this study, four male and one female patients whose ages vary between 20 days and 6 months were observed. The mean values of sodium, potassium and bicarbonate were found as $120 \pm 8.9 \mathrm{mmol} / \mathrm{l}, 7.18 \pm 0.98 \mathrm{mmol} / \mathrm{l}, 13.9 \pm 6.19$, respectively for five patients. Renin and aldosterone levels were high in all patients. All patients had urinary tract infection with different urinary tract anomalies. These anomalies were unilateral ureterovesical junction (UVJ) obstruction in two patients, bilateral vesicoureteral reflux (VUR) with posterior urethral valve (PUV) in one patient, PUV with bilateral UVJ obstruction in one patient, and unilateral VUR in one patient. The electrolyte imbalance and metabolic acidosis improved after treatment with intravenous saline and appropriate antibiotic agents. Also, renin and aldosterone levels were decreased to normal limits after the aforementioned treatments.
In this study, our aim was to increase awareness for type 1 secondary PHA among pediatricians. Type 1 secondary PHA should be considered in the presence of hyponatremia, hyperkalemia and metabolic acidosis in infants with urinary tract infections and/or urinary tract anomalies. Also, electrolyte and blood gas monitoring should be done in infants with urinary tract infections.

\section{P562 NOCTURNAL ENURESIS IN CHILDREN: MEDICAL COMORBIDITIES}

${ }^{1}$ Pietro Ferrara*, ${ }^{2}$ Roberta Autuori, ${ }^{2}$ Alessandro Di Lucia, ${ }^{2}$ Flavia Dosa, ${ }^{2}$ Giulia Franceschini, ${ }^{2}$ Diletta Saitta, ${ }^{2}$ Michela Pulcino, ${ }^{2}$ Federica Di Ruscio, ${ }^{2}$ Margherita Zona, ${ }^{2}$ Daniela Grasso, ${ }^{2}$ Rossella Giordano, ${ }^{3}$ Massimo Pettoello-Mantovani. ${ }^{1}$ Institute of Pediatrics, Catholic University Medical School, Rome, Italy; ${ }^{2}$ Campus Bio-Medico University, Rome, Italy; ${ }^{3}$ Department of Pediatrics, Scientific Institute Casa Sollievo della Sofferenza, University of Foggia, Foggia, Italy

\subsection{6/archdischild-2019-epa.896}

Objective To evaluate the characteristics of patients with NE. Design It is a cross-sectional study.

Setting During the physical examination, we sought signs and symptoms of voiding disorders and comorbidities with precise questions posed to the patients and their parents. Further, we assessed family history and behavioral characteristics of patients. Analyses were conducted using the Microsoft Excel 2016 program. We used descriptive statistics to describe patients' information.

Patients We recruited 403 children with Noctunal Enuresis (NE) at the Services of Pediatrics, Campus Bio-Medico University Hospital of Rome between June 2013 and July 2018. We rejected 2 children respectively with a renal agenesis and a chromosomopathy and we enrolled 401 patients, 101 girls (25.2\%) and 300 boys (74.8\%), aged 5-16 years; mean age at first visit $8.8 \pm 2.44$ years. Of the 401 eligible patients, we counted $327(81.5 \%)$ patients with monosymptomatic NE (MNE) and $74(18.5 \%)$ patients with non-monosymptomatic MNE (N-MNE). In the group of MNE children, 242 (74\%) children were boys with mean age at first examination 9.1 years and $85(26 \%)$ were girls with mean age at first examination 7.8 years. In the group of N-MNE children, 58 (78.4\%) children were boys with mean age at first examination 9 years and $16(21.6 \%)$ were girls with mean age at first examination 8.4 years. Among the children with MNE, 322 (98.5\%) had PMNE and 5 (1.5\%) were diagnosed with SMNE.

\section{Interventions Not applicable}

Main outcome measures Signs and symptoms of voiding disorders, comorbidities, family history of NE, behavioral characteristics and school achievement.

Results We noticed that heredity, parasomnias, urogenital abnormalities, constipation and innocence heart murmur are correlated to NE. Notably, 53.6\% (67/125 children) had paternal heredity, $46.4 \%(58 / 125)$ had maternal heredity; $11.2 \%$ $(14 / 125)$ had both paternal and maternal inheritance. 12/401 (3\%) had sleep apnea, 95/401 (23.7\%) had somniloquy and $59 / 401(14.7 \%)$ had bruxism. Children suffered from headaches were 21/401 (5.2\%): $112(27.3 \%)$ had family history of headache. $47 / 300$ boys $(15.7 \%)$ had urogenital-abnormalities: 28/47 (59.6\%) children had balanopreputial adhesions. Cutaneous manifestations of spinal dysraphism such as pilonidal dimple, single and deflected intergluteal cleft or double intergluteal cleft were found in 21/401 (5.2\%) patients. Encopresis 